\title{
Association of Depressive Symptoms with Hippocampal Volume in 1936 Adults
}

\author{
E Sherwood Brown ${ }^{* \prime}$, Carroll W Hughes', Roderick McColl ${ }^{2}$, Ronald Peshock ${ }^{2}$, Kevin S King ${ }^{2}$ and \\ A John Rush ${ }^{3}$ \\ 'Department of Psychiatry, The University of Texas Southwestern Medical Center, Dallas, TX, USA; ${ }^{2}$ Department of Radiology, \\ The University of Texas Southwestern Medical Center, Dallas, TX, USA; ${ }^{3}$ Duke-NUS, Singapore, Singapore
}

\begin{abstract}
Hippocampal atrophy is reported in major depressive disorder (MDD). However, sample sizes were generally modest, and participant characteristics, including age, differed between studies. This study used a community sample to examine relationships between current depressive symptom severity and hippocampal volume across the adult lifespan. A total of 1936 adults with magnetic resonance images of the brain and Quick Inventory of Depressive Symptomatology Self-Report (QIDS-SR) scores were included. Brain volumes were quantified using the FSL program. Multiple linear regressions were performed using left, right, and total hippocampal volume as criterion variables, and predictor variables of QIDS-SR total, total brain volume, age, gender, education, psychotropic medications, alcohol use, and race/ethnicity. Post hoc analyses were conducted in participants with QIDS-SR scores $\geqslant 1$ I (moderate or greater depressive symptom severity) and $<I I$, and older and younger adults. In the primary analysis (sample as a whole) QIDS-SR was inversely associated with total hippocampal volume $(b=-0.044, p=0.032,(\mathrm{Cl}-0.019$ to $-0.00 \mathrm{I}))$ but not with left or right hippocampal volume evaluated individually. In participants with QIDS-SR scores of < I I, hippocampal volumes were not associated with QIDS-SR scores. In those with QIDS-SR scores $\geqslant 1$ I total, right, and left hippocampal volumes were modestly, but significantly, associated with QIDS-SR scores. The association between QIDS-SR scores and the hippocampal volume was much stronger in older persons. Findings suggest smaller hippocampal volumes among those with greater reported depressive symptom severity —an association that is strongest in people with at least moderate depressive symptom levels.
\end{abstract}

Neuropsychopharmacology (2014) 39, 770-779; doi:I0.1038/npp.20 I3.27I; published online I3 November 20I3

Keywords: hippocampus; total brain volume; mood; quick inventory of depressive symptomatology; neurodegeneration

\section{INTRODUCTION}

Smaller hippocampal volume in people with major depressive disorder (MDD), as compared with healthy controls, has been reported in some but not all studies (Arnone et al, 2012a; Campbell et al, 2004; Kempton et al, 2011; McKinnon et al, 2009; Videbech and Ravnkilde, 2004). Differences in population characteristics, including age, may have contributed to disparate outcomes. The inconsistent findings from prior studies have led some to question the strength of the evidence supporting hippocampal atrophy in MDD (Fink, 2011). Hippocampal volume reduction in MDD is a potentially important finding for several reasons. First, the hippocampus is involved in declarative memory processes (Eichenbaum et al, 1992). Declarative memory appears to be impaired, and associated with hippocampal volume, in patients with MDD (Clark et al, 2009; Turner and Lloyd,

*Correspondence: Dr ES Brown, Department of Psychiatry, The University of Texas Southwestern Medical Center, 5323 Harry Hines Blvd. MC 8849, Dallas, TX 75390-8849, USA, Tel: + I 2146456950 , Fax: + I 214645 695I, E-mail: sherwood.brown@utsouthwestern.edu Received I July 2013; revised 4 September 2013; accepted 14 September 2013; accepted article preview online 4 October 2013
2003). Second, the hippocampus is part of a larger neural circuit that includes limbic structures and the medial prefrontal cortex that may be central to the affective, emotional, and cognitive features of MDD (Clark et al, 2009; Drevets et al, 2008). Third, hippocampal volume may be associated with treatment response in MDD. Smaller hippocampal volume appears to be associated with a poorer response to antidepressants (Hsieh et al, 2002; Sheline et al, 2012; Vakili et al, 2000).

The etiology of hippocampal volume reduction in persons with MDD is not clear. Depressive episodes may cause hippocampal atrophy. In support of this idea are data suggesting a relationship between hippocampal volume and length of lifetime depression (Sheline et al, 1996). Cumulative stress and adversity are associated with changes in some brain regions (Ansell et al, 2012). Corticosteroid excess is associated with hippocampal atrophy in animal models and humans (Brown et al, 2004). Therefore, cortisol elevation during depressive episodes is a possible mechanism for hippocampal volume reduction in MDD. However, a report found that current cortisol levels did not significantly mediate the relationship between hippocampal volume and depression (Gerritsen et al, 2011). Glutamatergic pathways could also contribute to hippocampal changes in persons 
with MDD. Dysregulation of genes involved in glutamatemediated neuronal and synaptic plasticity has been reported in postmortem hippocampal slices from depressed patients (Duric et al, 2013). Genetic factors may also have a role. Homozygosity for the L allele of the 5-HTTLPR biallelic polymorphism is associated with smaller hippocampal volume in patients with MDD but not controls (Frodl et al, 2004). Increased cellular density is another possible mechanism. A postmortem study found cellular changes including increased packing density of glia, pyramidal neurons, and granule cell neurons as well as differential shrinkage of frozen sections of the hippocampus, consistent with a reduction in water content, in patients with MDD (Stockmeier et al, 2004). Inflammation is an emerging area of interest in depression research (Eyre and Baune, 2012). Elevated levels of inflammatory biomarkers are associated with smaller hippocampal volumes in patients with MDD (Frodl et al, 2012).

Alternatively, rather than being a consequence of MDD, smaller hippocampal volume could potentially be a risk factor for the development of MDD. In support of this idea, reduced hippocampal volume has been reported in healthy girls at high risk for MDD based on family history (Chen et al, 2010). Some data suggest that small hippocampus may be a risk factor for post-traumatic stress disorder (Gilbertson et al, 2002), a stress-related disorder can cooccur with MDD.

A limitation of many studies of hippocampal volume in MDD has been small sample sizes. A solution to this problem has been to combine the data in meta-analyses. The meta-analyses reported, to date, have suggested that people with MDD have smaller mean hippocampal volume than non-depressed controls (Arnone et al, 2012a; Campbell et al, 2004; Kempton et al, 2011; McKinnon et al, 2009; Videbech and Ravnkilde, 2004). Meta-analysis helps achieve sufficient numbers for statistical significance but it does not address problems with selection bias inherent to casecontrol studies. Meta-analysis also depends on the ability to generalize findings from underlying heterogeneous studies. Differences in depression definition and method of assessment, magnetic resonance imaging (MRI) techniques, nature of control groups, age, gender, control for total brain volume or intracranial volume, and education levels of the participants have varied greatly between studies.

Inconsistent findings have been reported on age and genders effects on hippocampal volume in MDD. Frodl et al (2002) reported greater hippocampal volume reduction in men than in women with first episode MDD. However, a meta-analysis did not find a gender effect (Videbech and Ravnkilde, 2004). This same meta-analysis did not find an impact of age on hippocampal volume in MDD. However, a more recent meta-analysis reported greater hippocampal volume reduction in middle aged adults with MDD than in older or younger adults (McKinnon et al, 2009).

The current study examines the relationship between hippocampal volume and current depressive symptom severity in a population-based sample of 1936 adults participating in a large community-based research study. We hypothesized that current depressive symptom severity would be inversely associated with hippocampal volume. In addition, we used the large sample to explore age and gender effects.

\section{MATERIALS AND METHODS}

\section{Participants and Assessments}

The study population was obtained from the Dallas Heart Study (DHS), a multiethnic cohort of Dallas County English or Spanish speaking adult residents used to examine cardiovascular disease and collect data for future studies. The details of the participant selection process and the study design have been previously described (Neeland et al, 2012; Victor et al, 2004).

The DHS intentionally oversampled African-Americans to comprise $\sim 50 \%$ of the participants in order to explore cardiovascular disease risk factors in this subpopulation. All participants signed written informed consents approved by The University of Texas Southwestern Medical Center Institutional Review Board. The first phase of the study (DHS-1) did not assess either depressive symptoms or brain volumes. The data in the current report are from a second phase of the study (DHS-2). The DHS-2 sample had a slightly higher proportion of women and Caucasians than in the original DHS-1 population due to differences in attrition following DHS-1. The participants in DHS-2 included people who had participated in DHS-1as well as some family members and/or spouses of DHS-1 participants. DHS-2 was conducted from September 2007 to December 2009. For more information about DHS-2 please see these references (King et al, 2013; Kozlitina and Garcia, 2012; Lucarelli et al, 2013).

Extensive information, including demographic characteristics, was obtained as part of the study. Race and ethnicity were determined through self-identification and the categories included: African-American, Caucasian (non-Hispanic White), Hispanic, and Other (Native American, Alaska Native, Asian, Pacific Islander, and East Indian). DHS-2 collected MRI scans of the brain and other organ systems.

The 16-item Quick Inventory of Depressive Symptomatology Self-Report (QIDS-SR) was also administered at DHS-2. The QIDS-SR is a 16-item, 0-3, patient-rated assessment of depressive symptom severity over the past 7 days (Rush et al, 2000; Rush et al, 2003; Trivedi et al, 2004). The QIDS-SR assesses the nine symptom domains that define MDD. The internal consistency of the QIDS-SR (Chronbach's alpha $=0.86$ ) is comparable to that of the 17item Hamilton Rating Scale for Depression $\left(\mathrm{HAMD}_{17}\right)$ (Rush et al, 2003). Scores on the QIDS-SR correlate highly with those of the longer 30-item IDS-SR $30(r=0.91)$ and the HAMD $_{17}(r=0.85)$ (Rush et al, 1996). QIDS-SR total score multiplied by 1.3 is approximately equivalent to the HAMD $_{17}$ total score (Rush et al, 2003). As in the National Comorbidity Survey Replication (Kessler et al, 2003), transformation rules were used to convert QIDS-SR scores into depressive symptom severity categories mapped to conventional HAMD ranges of none (0-5), mild (6-10), moderate (11-15), severe (16-20), and very severe $(21+)$ (Rush et al, 2003). For more information about the psychometric properties and use of the QIDS-SR see www.ids-qids.org.

\section{Neuroimaging}

Both MP-RAGE and FLAIR images were collected. All images were acquired on the same 3T MRI scanner 
(Achieva, Philips Medical Systems, Best, the Netherlands). The images were taken in axial orientation from the vertex of the skull to the foramen magnum. The 3D MP-RAGE images were acquired with $\mathrm{TR} / \mathrm{TE}=9.6 / 5.8 \mathrm{msec}$, flip angle $=12$ degrees, SENSE factor $=2$, field of view $(\mathrm{FOV})=260 \times 260 \mathrm{~mm}, 2 \mathrm{~mm}$ slices spaced at $1 \mathrm{~mm}$ centers, Rows $\times$ Cols $\times$ Slices $=288 \times 288 \times 140$, and voxel size of $1 \times 0.9 \times 0.9 \mathrm{~mm}$ (Hulsey et al, 2012).

MRI quantification was performed using the freely available FMRIB software library, FSL (fsl.fmrib.ox.ac.uk). Volumes of the left and right hippocampus were derived from 3D-MP-RAGE sequences using the FSL tool FIRST (fsl.fmrib.ox.ac.uk/fsl/fslwiki/FIRST) (Patenaude et al, 2011), which is a model-based segmentation and registration tool and which can segment subcortical structures, including the hippocampi, automatically. Total brain volume (gray matter plus white matter) was also obtained. Volumetric data were collected using the FSL routine called fslstats. Volumes of the left and right hippocampus, along with other cortical and subcortical structures not reported here, were derived from MP-RAGE sequences. For more information on the imaging methods in DHS see Hulsey et al (2012). Scans identified by an error code produced by the software or identified by review of outliers were inspected. Scans containing artifacts, encephalomalacia, or other abnormalities were excluded from the analysis. Individuals who were excluded from the MRI included people with a history of brain surgery, metal fragments, pacemakers, implantable cardiodefribrillators, cochlear implants, spinal cord stimulators, or other internal electrical devices. Individuals who were pregnant or had jobs that could have exposed them to metal fragments were also excluded from the MRIs. A total of 2082 participants underwent brain MR imaging. Thirty-seven were excluded for self-reported stroke. Images of outliers as found by Robust Minimum Covariance Distance analysis of brain segments (Lucarelli et al, 2013), individuals flagged for exclusion in previous DHS-2MR imaging brain studies, and individuals who had error flags generated during automated analysis were reviewed by a neuroradiologist (KSK). On MR imaging review, 70 individuals with major structural defects (such as corpus callosum agenesis, imaging evidence of stroke, and hydrocephalus) or image-acquisition errors (such as metal and motion artifacts, and other noise) were excluded. In total, 107 individuals were excluded from subsequent analysis. The segmentation failure rate of the overall sample was $1.4 \%$. In the current report, participants were also excluded if they had missing data for any of the other predictor or criterion variables tested resulting in 1936 participants used in these analyses.

\section{Statistical Analysis \\ Multiple linear regressions were performed using SPSS version 20.0 (IBM SPSS Statistics) with left, right, and total hippocampal volume $(\mathrm{ml})$ as criterion variables, and predictor variables of QIDS-SR total score, total brain volume (ml), age (years), gender (male, female), education (years), psychotropic medications (antidepressants, anti- psychotics, anticonvulsants, anxiolytics, hypnotics, and stimulants), alcohol use (current drinking, recent abstainer, and lifetime abstainer), and race/ethnicity (Caucasian,}

African-American, Hispanic, and Other). In addition to the above analysis in the entire sample, post hoc linear regressions were performed, using the same criterion and predictor variables as above in participants with QIDS-SR scores of $<11$ and $\geqslant 11$ (moderate depressive symptom severity or greater). A QIDS-SR score of 11 is approximately equivalent to a $\mathrm{HAMD}_{17}$ of about $14-15$, which is potentially consistent with at least mild MDD (Rush et al, 2003). These QIDS-SR scores were used to define depression relapse in the large Sequenced Treatment Alternatives to Relieve Depression $\left(\mathrm{STAR}^{\star} \mathrm{D}\right)$ study (Rush et al, 2006a). Age $\times$ QIDS-SR and gender $\times$ QIDS-SR interactions were also explored in the entire sample and in those with QIDS-SR scores $\geqslant 11$.

\section{RESULTS}

The demographic characteristics of the participants are in Table 1. A total of $58.5 \%$ were women and $46.2 \%$ were African-American. The mean $( \pm S D)$ age was $49.7 \pm 10.6$ years, and mean education level was $12.8 \pm 2.3$ years. Mean total QIDS-SR score was $5.1 \pm 3.8$ (range $0-24$ ). A total of $9.9 \%$ of the entire sample and $20.3 \%$ of those with at least moderate depressive symptom severity (QIDS-SR $\geqslant 11$ ) were currently taking antidepressants. Participants with missing

Table I Demographic Features, $N=1936$

\begin{tabular}{|c|c|c|}
\hline Characteristics & $N$ & $\%$ \\
\hline \multicolumn{3}{|l|}{ Sex } \\
\hline Male & 804 & 41.5 \\
\hline Female & 1132 & 58.5 \\
\hline \multicolumn{3}{|l|}{ Race } \\
\hline Caucasian & 702 & 37.2 \\
\hline Hispanic & 276 & 14.3 \\
\hline African-American & 895 & 46.2 \\
\hline Other & 40 & 2.1 \\
\hline \multicolumn{3}{|l|}{ Concomitant medication } \\
\hline Stimulants & 33 & 1.7 \\
\hline Anticonvulsants & 59 & 3.0 \\
\hline Antidepressants & 192 & 9.9 \\
\hline Antipsychotics & 28 & 1.4 \\
\hline Anxiolytics & 90 & 4.6 \\
\hline \multirow[t]{2}{*}{ Hypnotics } & 78 & 4.0 \\
\hline & Mean & SD \\
\hline Age (years) & 49.7 & 10.6 \\
\hline Education (years) & 12.8 & 2.3 \\
\hline QIDS-SR score & 5.1 & 3.8 \\
\hline \multicolumn{3}{|l|}{ Volumes (ml) } \\
\hline Total brain & 916.5 & 105.0 \\
\hline Total hippocampus & 6.8 & 0.8 \\
\hline Left hippocampus & 3.3 & 0.5 \\
\hline Right hippocampus & 3.4 & 0.5 \\
\hline
\end{tabular}


data and, therefore, not included in the analysis, were demographically similar $(60.1 \%$ women, $56.4 \%$ AfricanAmerican, $12.0 \%$ taking antidepressants, age 50.2 \pm 11.9 , and QIDS-SR 5.6 \pm 4.2 ) to those included in the analysis.

Results of multiple linear regression analyses, using hippocampal volume as the criterion variable, are presented in Table 2. After controlling for demographic features and total brain volume, total hippocampal volume was inversely associated with total QIDS-SR score $(b=-0.044, p=0.032$ $(\mathrm{CI}-0.019$ to 0.001$))$. Left $(b=-0.036, \quad p=0.085$ $(\mathrm{CI}-0.009$ to 0.001$))$ and right $(b=-0.029, p=0.167$ (CI -0.009 to 0.002$)$ ) hippocampal volumes when evaluated individually did not reach statistical significance. Total brain volume and race/ethnicity were also significantly related to hippocampal volume in these analyses. Gender was significantly related to right and total, but not left, hippocampal volume. An independent association of age with hippocampal volume only reached significance on the right. The variance inflation factor (VIF) a measure of multicollinearity ranged from 1.049 to 1.651 for the predictor variables, including 1.129 for the predictor variable of interest (QIDS-SR scores). Because these values were modest (Pan and Jackson, 2008), predictor variables were not removed or centered to manage high intercorrelation. To examine age and gender effects on the relationship between depression and hippocampal volume, we explored age $\times$ QIDS-SR $(b=0.41, p=0.674)$ and gender $\times$ QIDS-SR $(b=-0.031, p=0.705)$ interactions both of which were nonsignificant.

Given the relatively modest associations between the severity of current depressive symptoms and hippocampal volume in this sample, and in light of the many studies suggesting a reduction in hippocampal volume in people with a diagnosis of MDD, post hoc analyses (including the same predictor variables as in the primary analysis) in those with QIDS-SR scores $\geqslant 11$ and $<11$ were conducted to see whether stronger associations were observed in those with at least moderate levels of depressive symptom severity that might be consistent with current MDD (Table 3). Scatter plots of hippocampal volumes $v s$ QIDS-SR scores are in Figure 1. In those with QIDS-SR scores $<11$ no significant relationships between hippocampal volumes and QIDS-SR scores were observed. However, in those with QIDS-SR scores $\geqslant 11$ total $(b=-0.184, p=0.005,(\mathrm{CI}-0.092$ to $0.016)$, left $(b=-0.135, p=0.042$ (CI -0.042 to 0.001$))$, and right $(b=-0.134, p=0.049(\mathrm{CI}-0.044$ to 0.000$))$ hippocampal volumes were significantly related to QIDS-SR scores. Other predictor variables such as race/ethnicity, gender, and age were no longer significantly associated with hippocampal volume in the group with higher levels of depressive symptom severity.

We examined age $\times$ QIDS-SR and gender $\times$ QIDS-SR interaction terms in the regression model of total hippocampal volume in those with QIDS-SR scores $\geqslant 11$. Gender $\times$ QIDS-SR interaction was nonsignificant $(p=0.058)$. However, a significant age $\times$ QIDS-SR interaction was observed $(p=0.032)$. Based on a meta-analysis that found greater hippocampal volume reduction in older and younger adults than middle aged adults with MDD (McKinnon et al, 2009), we conducted linear regressions in ages $<40,40-59$, and 60 years and above, in those with QIDS-SR scores $\geqslant 11$ using total hippocampal volume as the criterion variable and the same predictor variables as in the other analyses. The standardized coefficients and significance for the QIDS-SR increased with age (age $<40, n=36$, $b=-0.129, p=0.268$; age $40-59, n=122, b=-0.173$, $p=0.480$; age $60+, n=25, b=-0.651, p<0.001)$.

\section{DISCUSSION}

The findings suggest that current depressive symptom severity is negatively associated with total hippocampal volume in a population-based sample. Prior research has generally used an MDD diagnosis when assessing hippocampal volume. This study suggests a relationship between the level of current depressive symptom severity and size of the hippocampus in a sample that includes participants with and without symptom severity that typifies a major depressive episode. However, it is important to note that the observed inverse relationship between QIDS-SR scores and hippocampal volume was modest and only reached significance for total hippocampal volume, not left, or right volumes.

Unlike the current study, most prior studies examining the relationship between hippocampal volume and depression have used participants with a diagnosis of syndromal MDD based on clinical criteria and a non-depressed control group. Therefore, we conducted a post hoc analysis to determine whether the relationship between hippocampal volume and depressive symptom severity was stronger in those with at least a moderate level of depressive symptom severity based on the QIDS-SR. In participants with lower levels of depressive symptom severity, no significant relationships between QIDS-SR scores and hippocampal volumes were observed. However, in participants with more clinically significant levels of depressive symptoms, the relationships between QIDS-SR scores and total, right, and left hippocampal volume were significant. The standardized coefficients (a measure of SD change in a criterion variable based on SD change in the predictor variable) were approximately nine times larger in the group with higher QIDS-SR scores as compared with those with lower QIDSSR scores (Table 3), and four times larger than in the sample as a whole (Table 2). In addition, other independent variables, with the exception of total brain volume, lost significance in the group with higher QIDS-SR scores. These data are potentially consistent with the idea of a threshold level of depression at which hippocampal volume is related to the levels of depression severity. Furthermore, the data suggest that relatively mild depressive symptoms are not associated with hippocampal volume differences. A recent report by Spalletta et al, (2013) examined the relationship between hippocampal volume and Beck Depression Inventory (BDI ) scores in 102 participant free of psychiatric illness. BDI scores were consistent with minimal to mild depression. A significant correlation was observed between hippocampal volume and BDI score in men but not in women. These findings differ from findings in our participants with lower QIDS-SR scores (Table 3). The differences could be due to different participant characteristics, differences in measurement of brain volumes, or differences in the depression scales.

Before the current report, few large studies have examined the relationship between depression and 
Table 2 Linear Regression Analyses of Total, Left, and Right Hippocampal Volume $(n=1936)$

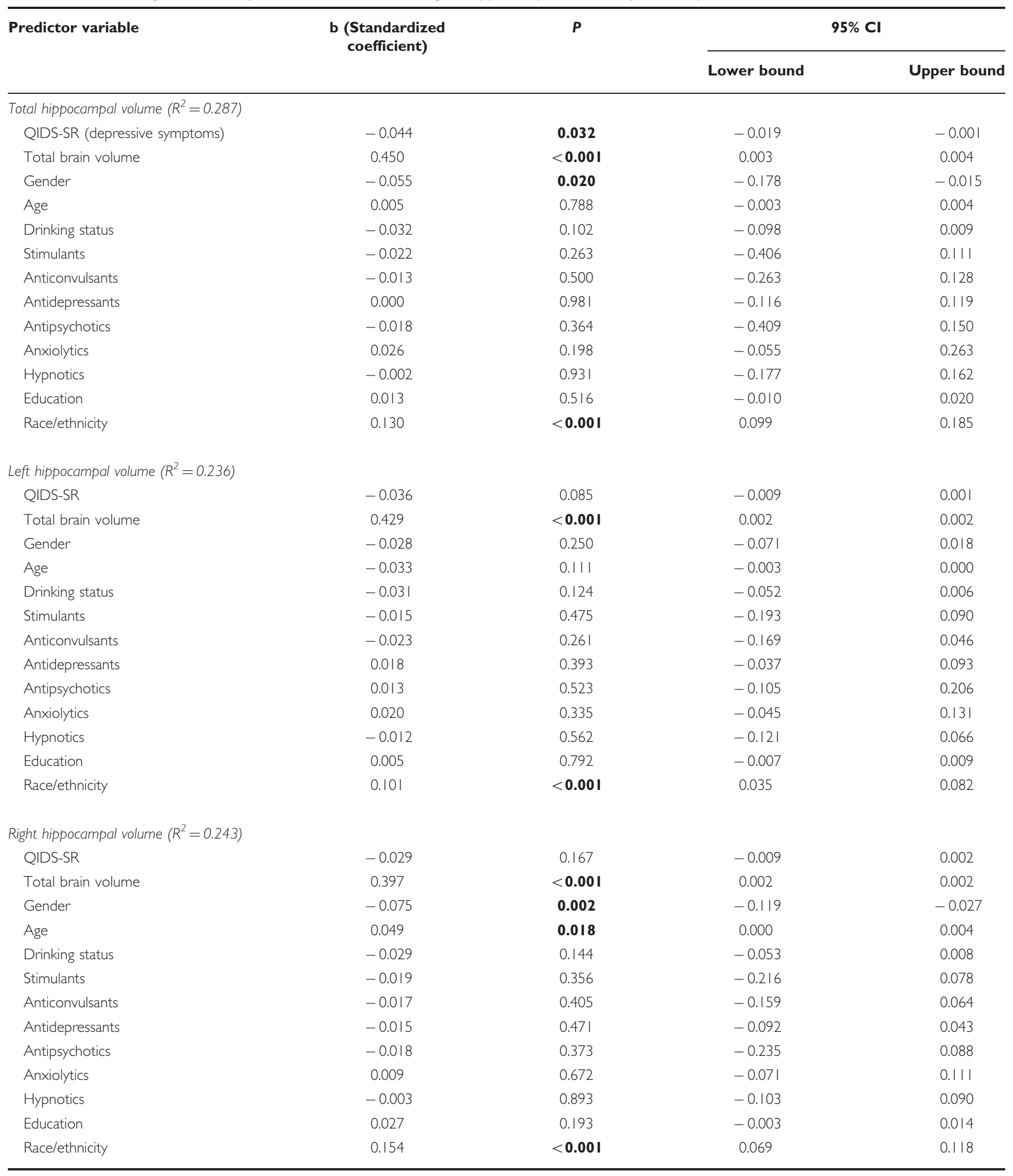

Bold values denote statistical significance.

hippocampal volume. In one such study, Gerritsen et al (2011) examined depression and hippocampal volume in people with atherosclerosis $(N=636)$. They reported that a lifetime history of depression and current depression were associated with approximately a $1.7 \%(p<0.05$ on left but not right) and $2.3 \%(p=\mathrm{NS})$ smaller hippocampal volume, respectively. However, current depressive symptom severity, as assessed by the Patient Health Questionnaire 
Table 3 Linear Regression Analyses of Total, Left, and Right Hippocampal Volume By QIDS-SR score < I vs $\geqslant$ | I

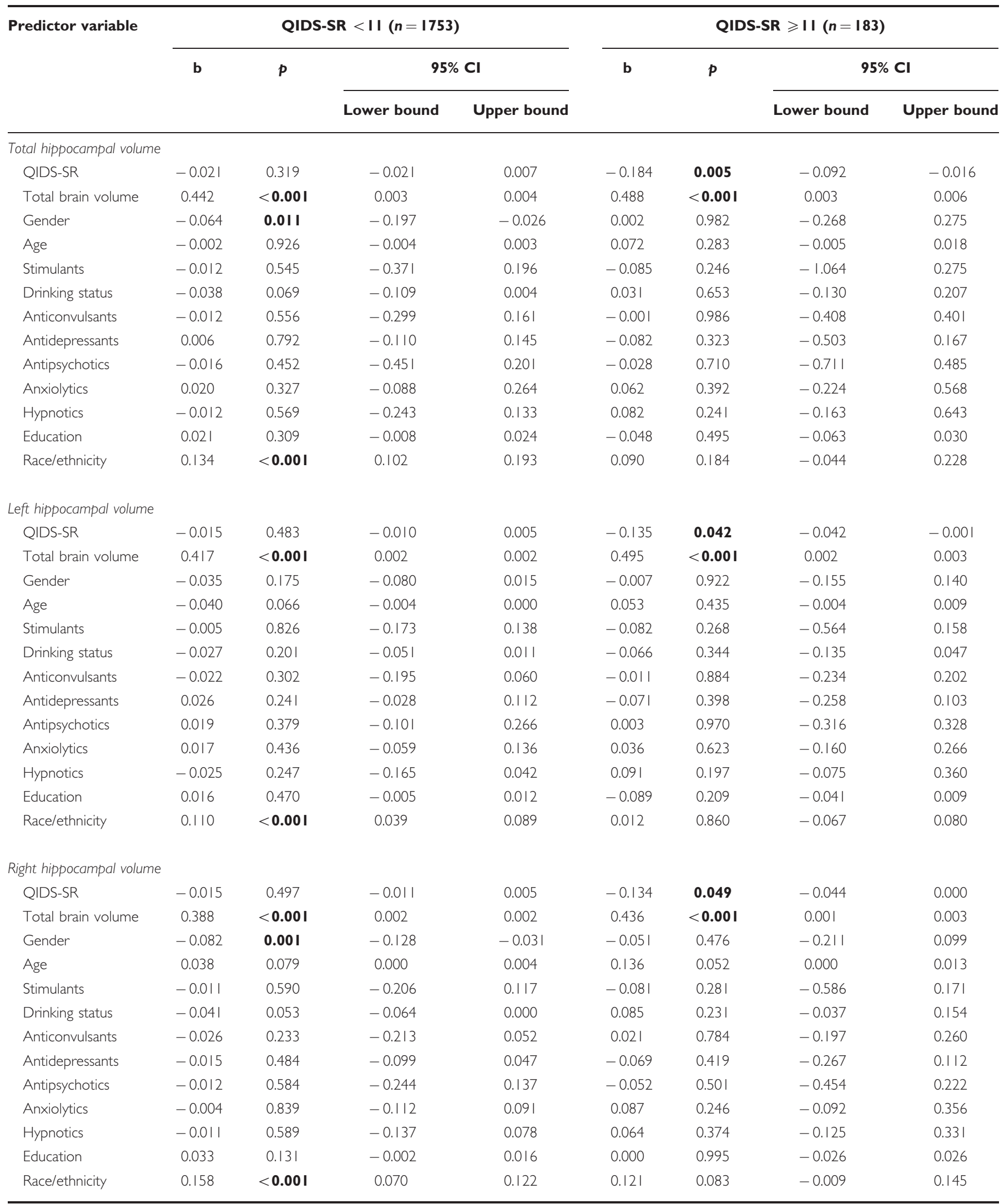

Bold values denote statistical significance. 

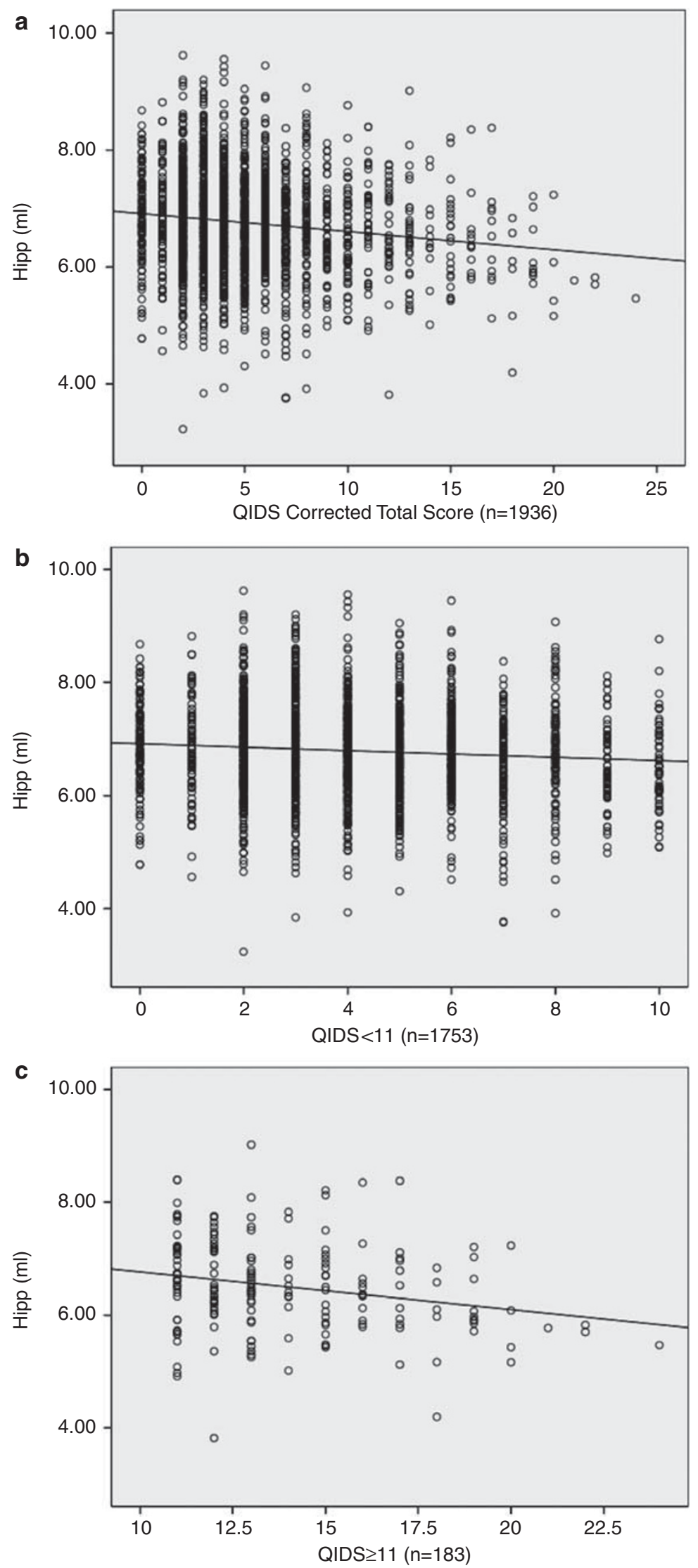

Figure I Scatterplots of total hippocampal volume vs Quick Inventory of Depressive Symptomatology Self-Report (QIDS-SR) scores in (a) the total sample, (b) participants with QIDS-SR scores <II and (c) participants with QIDS-SR scores $\geqslant$ II. 
(PHQ-9) (Kroenke et al, 2001), was not significantly associated with the hippocampal volume. Thus, our current report found a more robust association between depressive symptom severity and hippocampal volume than the report by Gerritsen et al (2011). This difference may be due to the larger sample size in the current report and greater representation of women (58vs<20\%). Some reports suggest a greater decline in hippocampal volume with age in men than in women (Pruessner et al, 2001). Therefore, a sample primarily consisting of men could show greater agerelated hippocampal atrophy in general, and, therefore, less difference with depression. Because depression is more common in women than in men, a sample consisting of mostly men might also have fewer participants with elevated depressive symptoms scores. By design, the DHS was racially and ethnically diverse and oversampled for African-Americans. To our knowledge, one large study $(N>600)$ in an ethnically diverse sample has been previously reported. Geerlings et al (2012) examined hippocampal volume in a community sample $(N=630$, 29\% Caucasian, 34\% African-American, 36\% Hispanic, and $2 \%$ Other) of older persons (mean age 80 years). They reported that participants with current depression, defined as a Center for Epidemiologic Studies-Depression Scale score $\geqslant 4$ or current antidepressant use, had smaller hippocampal volumes.

Studies in both living depressed patients (Sheline et al, 2003) and postmortem analyses (Boldrini et al, 2013) suggest that antidepressants may modify the association between hippocampal volume and depression. However, in the current report none of the analyses suggested a relationship between hippocampal volume and antidepressant, or other psychotropic medication use. This difference may potentially be explained by two factors. First, antidepressant use was not common. Thus, we may not have the power to detect effects of the antidepressants on hippocampal volume. Second, given the design of the study, we do not know how long participants had been taking an antidepressant. If antidepressant treatment was initiated shortly before the MRI was obtained then one might expect little effect of the antidepressant on the hippocampal volume.

Our findings suggest that the relationship between depressive symptom severity and hippocampal volume may increase in older adults. The standardized coefficient was five times greater in those age 60 years and over as compared with those under age 40. These findings differ somewhat from the findings of a meta-analysis that reported the greatest reduction in hippocampal volume was observed in middle age adults (McKinnon et al, 2009). These differences may be explained by differences in the study methods and design. Our analysis examined relationships between current depressive symptom severity and hippocampal volume in different age groups rather than examining differences in volume compared with controls in participants with MDD. In addition, the age of onset of depression and illness duration varied among the studies in the meta-analysis and is not known in our study. Thus, the participant characteristics may be quite different. Because the hippocampus is involved in memory, our findings are potentially consistent with the greater deficits in memory performance in older $v s$ younger persons with MDD (Thomas et al, 2009).
Strengths of the study include the large population-based sample and racial/ethnic diversity, which increase the generalizability of the findings. The size of the sample allowed for an analysis of the impact of depressive symptom severity as well as gender and age. Our analyses controlled for total brain volume (white matter plus gray matter) minimizing the effects of more generalized brain atrophy beyond the hippocampus. Although the total sample size was very large, the subgroups that were analyzed were much smaller which may increase the risk of type II errors. In addition, the study controlled for medication use, alcohol use, education, and other variables that might influence the relationship between depression and hippocampal volume. Another potential strength is the use of an automated method to derive hippocampal volumes that results in excellent reproducibility (Lucarelli et al, 2012; Nugent et al, 2012) and avoids a left-right bias that may be inherent with manual segmentation (Maltbie et al, 2012).

The study has several limitations. The QIDS-SR is a selfrated instrument that assesses current depressive symptom severity. A strength of the QIDS-SR is its ability to assess current depressive symptoms. The QIDS-SR assesses core symptoms of MDD in the DSM-IV-TR and is strongly associated with scores on clinician-rated depression instruments (Rush et al, 2003) and structured diagnostic interviews (Bernstein et al, 2009; Doraiswamy et al, 2010), and has been used in large clinical studies (Kessler et al, 2003; Rush et al, 2006b; Trivedi et al, 2006). However, if the hippocampal volume changes occur over long periods then the study is limited by only comparing a slowly changing brain change with a current measure of depression. Because of the study design, limited information was available on the neurological histories of the participants. However, we were able to exclude participants with a known history of stroke, a condition that might directly impact brain volumes. Although the findings are somewhat mixed in terms of laterality, studies generally suggest that severe alcohol dependence is associated with reduced hippocampal volume (Beresford et al, 2006; Laakso et al, 2000; Le Berre et al, 2012; Ozsoy et al, 2013). To our knowledge, the degree of reversibility of hippocampal volume changes with alcohol dependence has not been investigated. The current report controlled for current alcohol use but lifetime alcohol use patterns were not available. Thus, we cannot rule out the possibility that past heavy alcohol use may have influenced the findings.

Given the cross-sectional nature of the study, it cannot address mechanisms, causality, or reversibility. As discussed in the introduction, numerous mechanisms might potentially result in hippocampal changes with depression. Changes in the hippocampus could either be a result of biological changes with depression or be a pre-existing risk factor for the development or chronicity of depression. Data are mixed on whether hippocampal volume changes in depression are reversible. The smaller hippocampal volumes in older patients with past, but not current, MDD in the report by Sheline et al (1996) might suggest that volume reduction is either irreversible or resolves very slowly. However, a recent report by Arnone et al (2012b) observed hippocampal gray matter reduction in patients with current, but not remitted depression, as compared with controls. These findings are potentially consistent with a reversible process. 
In summary, elevated QIDS-SR scores were associated with decreased total hippocampal volumes in a populationbased sample of 1936 participants. The relationship between depressive symptom severity and hippocampal volume was much stronger in those with at least moderate levels of depressive symptoms. In those participants with at least moderate depressive symptom severity the strength of the relationship between depressive symptom severity and hippocampal volume increased with age.

\section{FUNDING AND DISCLOSURE}

Dr Brown would like to disclose research support from NIMH, NIDA, NHLBI, Stanley Medical Research Institute, Forest Laboratories, and Sunovion Pharmaceuticals. Dr Hughes is a consultant and on the scientific advisory board for BioBehavioral Diagnostics and Naturally Slim, and has funding from NIMH. Dr Peshock serves as an advisor for Philips Medical Systems. Dr King reports support from NIH KL2 TR000453-06 Clinical and Translational Science Award. Dr Rush has received consulting fees from Otsuka Pharmaceutical Brain Resource and $\mathrm{H}$ Lundbeck $\mathrm{A} / \mathrm{S}$, speaker fees from Singapore College of Family Physicians, royalties from Guilford Publications and the University of Texas Southwestern Medical Center, a travel grant from CINP, and research support from Duke-National University of Singapore. Dr McColl reports no biomedical financial interests or potential conflicts of interest. Supported in part by grant UL1TR000451 from the National Center for Advancing Translational Sciences, National Institutes of Health.

\section{REFERENCES}

Ansell EB, Rando K, Tuit K, Guarnaccia J, Sinha R (2012). Cumulative adversity and smaller gray matter volume in medial prefrontal, anterior cingulate, and insula regions. Biol Psychiatry 72: 57-64.

Arnone D, McIntosh AM, Ebmeier KP, Munafo MR, Anderson IM (2012a). Magnetic resonance imaging studies in unipolar depression: systematic review and meta-regression analyses. Eur Neuropsychopharmacol 22: 1-16.

Arnone D, McKie S, Elliott R, Juhasz G, Thomas EJ, Downey D et al (2012b). State-dependent changes in hippocampal grey matter in depression. Mol Psychiatry (e-pub ahead of print).

Beresford TP, Arciniegas DB, Alfers J, Clapp L, Martin B, Du Y et al (2006). Hippocampus volume loss due to chronic heavy drinking. Alcohol Clin Exp Res 30: 1866-1870.

Bernstein IH, Wendt B, Nasr SJ, Rush AJ (2009). Screening for major depression in private practice. J Psychiatr Pract 15: 87-94.

Boldrini M, Santiago AN, Hen R, Dwork AJ, Rosoklija GB, Tamir H et al (2013). Hippocampal granule neuron number and dentate gyrus volume in antidepressant-treated and untreated major depression. Neuropsychopharmacology 38: 1068-1077.

Brown ES, Varghese FP, McEwen BS (2004). Association of depression with medical illness: does cortisol play a role? Biol Psychiatry 55: 1-9.

Campbell S, Marriott M, Nahmias C, MacQueen GM (2004). Lower hippocampal volume in patients suffering from depression: a meta-analysis. Am J Psychiatry 161: 598-607.

Chen MC, Hamilton JP, Gotlib IH (2010). Decreased hippocampal volume in healthy girls at risk of depression. Arch Gen Psychiatry 67: 270-276.
Clark L, Chamberlain SR, Sahakian BJ (2009). Neurocognitive mechanisms in depression: implications for treatment. Annu Rev Neurosci 32: 57-74.

Doraiswamy PM, Bernstein IH, Rush AJ, Kyutoku Y, Carmody TJ, Macleod L et al (2010). Diagnostic utility of the Quick Inventory of Depressive Symptomatology (QIDS-C16 and QIDS-SR16) in the elderly. Acta Psychiatr Scand 122: 226-234.

Drevets WC, Price JL, Furey ML (2008). Brain structural and functional abnormalities in mood disorders: implications for neurocircuitry models of depression. Brain Struct Funct 213: 93-118.

Duric V, Banasr M, Stockmeier CA, Simen AA, Newton SS, Overholser JC et al (2013). Altered expression of synapse and glutamate related genes in post-mortem hippocampus of depressed subjects. Int J Neuropsychopharmacol 16: 69-82.

Eichenbaum H, Otto T, Cohen NJ (1992). The hippocampus-what does it do? Behav Neural Biol 57: 2-36.

Eyre H, Baune BT (2012). Neuroplastic changes in depression: a role for the immune system. Psychoneuroendocrinology 37: 1397-1416.

Fink G (2011). Stress controversies: post-traumatic stress disorder, hippocampal volume, gastroduodenal ulceration*. J Neuroendocrinol 23: 107-117.

Frodl T, Carballedo A, Hughes MM, Saleh K, Fagan A, Skokauskas $\mathrm{N}$ et al (2012). Reduced expression of glucocorticoid-inducible genes GILZ and SGK-1: high IL-6 levels are associated with reduced hippocampal volumes in major depressive disorder. Translational Psychiatry 2: e88.

Frodl T, Meisenzahl EM, Zetzsche T, Born C, Groll C, Jager M et al (2002). Hippocampal changes in patients with a first episode of major depression. Am J Psychiatry 159: 1112-1118.

Frodl T, Meisenzahl EM, Zill P, Baghai T, Rujescu D, Leinsinger G et al (2004). Reduced hippocampal volumes associated with the long variant of the serotonin transporter polymorphism in major depression. Arch Gen Psychiatry 61: 177-183.

Geerlings MI, Brickman AM, Schupf N, Devanand DP, Luchsinger JA, Mayeux R et al (2012). Depressive symptoms, antidepressant use, and brain volumes on MRI in a populationbased cohort of old persons without dementia. J Alzheimers Dis 30: 75-82.

Gerritsen L, Comijs HC, van der Graaf Y, Knoops AJ, Penninx BW, Geerlings MI (2011). Depression, hypothalamic pituitary adrenal axis, and hippocampal and entorhinal cortex volumes-the SMART Medea study. Biol Psychiatry 70: 373-380.

Gilbertson MW, Shenton ME, Ciszewski A, Kasai K, Lasko NB, Orr SP et al (2002). Smaller hippocampal volume predicts pathologic vulnerability to psychological trauma. Nat Neurosci 5: 1242-1247.

Hsieh MH, McQuoid DR, Levy RM, Payne ME, MacFall JR, Steffens DC (2002). Hippocampal volume and antidepressant response in geriatric depression. Int J Geriatr Psychiatry 17: 519-525.

Hulsey KM, Gupta M, King KS, Peshock RM, Whittemore AR, McColl RW (2012). Automated quantification of white matter disease extent at $3 \mathrm{~T}$ : comparison with volumetric readings. J Magn Reson Imaging 36: 305-311.

Kempton MJ, Salvador Z, Munafo MR, Geddes JR, Simmons A, Frangou $S$ et al (2011). Structural neuroimaging studies in major depressive disorder. Meta-analysis and comparison with bipolar disorder. Arch Gen Psychiatry 68: 675-690.

Kessler RC, Berglund P, Demler O, Jin R, Koretz D, Merikangas KR et al (2003). The epidemiology of major depressive disorder: results from the National Comorbidity Survey Replication (NCSR). JAMA 289: 3095-3105.

King KS, Chen KX, Hulsey KM, McColl RW, Weiner MF, Nakonezny PA et al (2013). White matter hyperintensities: use of aortic arch pulse wave velocity to predict volume independent of other cardiovascular risk factors. Radiology 267: 709-717.

Kozlitina J, Garcia CK (2012). Red blood cell size is inversely associated with leukocyte telomere length in a large multi-ethnic population. PLoS One 7: e51046. 
Kroenke K, Spitzer RL, Williams JB (2001). The PHQ-9: validity of a brief depression severity measure. J Gen Intern Med 16: 606-613.

Laakso MP, Vaurio O, Savolainen L, Repo E, Soininen H, Aronen $\mathrm{HJ}$ et al (2000). A volumetric MRI study of the hippocampus in type 1 and 2 alcoholism. Behav Brain Res 109: 177-186.

Le Berre AP, Rauchs G, La Joie R, Mezenge F, Boudehent C, Vabret $\mathrm{F}$ et al (2012). Impaired decision-making and brain shrinkage in alcoholism. Eur Psychiatry (e-pub ahead of print).

Lucarelli RT, Peshock RM, McColl R, Hulsey K, Ayers C, Whittemore AR et al (2012). MR imaging of hippocampal asymmetry at $3 \mathrm{~T}$ in a multiethnic, population-based sample: Results from the Dallas Heart Study. AJNR Am J Neuroradiol 34: 752-757.

Lucarelli RT, Peshock RM, McColl R, Hulsey K, Ayers C, Whittemore AR et al (2013). MR imaging of hippocampal asymmetry at $3 \mathrm{~T}$ in a multiethnic, population-based sample: results from the Dallas Heart Study. AJNR Am J Neuroradiol 34: 752-757.

Maltbie E, Bhatt K, Paniagua B, Smith RG, Graves MM, Mosconi MW et al (2012). Asymmetric bias in user guided segmentations of brain structures. Neuroimage 59: 1315-1323.

McKinnon MC, Yucel K, Nazarov A, MacQueen GM (2009). A meta-analysis examining clinical predictors of hippocampal volume in patients with major depressive disorder. J Psychiatry Neurosci 34: 41-54.

Neeland IJ, Turer AT, Ayers CR, Powell-Wiley TM, Vega GL, Farzaneh-Far R et al (2012). Dysfunctional adiposity and the risk of prediabetes and type 2 diabetes in obese adults. JAMA 308: $1150-1159$.

Nugent AC, Luckenbaugh DA, Wood SE, Bogers W, Zarate CA Jr., Drevets WC (2012). Automated subcortical segmentation using FIRST: Test-retest reliability, interscanner reliability, and comparison to manual segmentation. Hum Brain Mapp 34: 2313-2329.

Ozsoy S, Durak AC, Esel E (2013). Hippocampal volumes and cognitive functions in adult alcoholic patients with adolescentonset. Alcohol 47: 9-14.

Pan Y, Jackson RT (2008). Ethnic difference in the relationship between acute inflammation and serum ferritin in US adult males. Epidemiol Infect 136: 421-431.

Patenaude B, Smith SM, Kennedy DN, Jenkinson M (2011). A Bayesian model of shape and appearance for subcortical brain segmentation. Neuroimage 56: 907-922.

Pruessner JC, Collins DL, Pruessner M, Evans AC (2001). Age and gender predict volume decline in the anterior and posterior hippocampus in early adulthood. J Neurosci 21: 194-200.

Rush A, Carmody T, PE R (2000). The Inventory of Depressive Symptomatology (IDS): Clinician (IDS-C) and self-report (IDS-SR) ratings of depressive symptoms. Int J Meth Psychiatr Res 9: 45-59.

Rush AJ, Gullion CM, Basco MR, Jarrett RB, Trivedi MH (1996). The Inventory of Depressive Symptomatology (IDS): psychometric properties. Psychol Med 26: 477-486.

Rush AJ, Trivedi MH, Ibrahim HM, Carmody TJ, Arnow B, Klein DN et al (2003). The 16-Item Quick Inventory of Depressive
Symptomatology (QIDS), clinician rating (QIDS-C), and selfreport (QIDS-SR): a psychometric evaluation in patients with chronic major depression. Biol Psychiatry 54: 573-583.

Rush AJ, Trivedi MH, Wisniewski SR, Nierenberg AA, Stewart JW, Warden D et al (2006a). Acute and longer-term outcomes in depressed outpatients requiring one or several treatment steps: a STAR ${ }^{\star D}$ report. Am J Psychiatry 163: 1905-1917.

Rush AJ, Trivedi MH, Wisniewski SR, Stewart JW, Nierenberg AA, Thase ME et al (2006b). Bupropion-SR, sertraline, or venlafaxine-XR after failure of SSRIs for depression. $N$ Engl J Med 354: $1231-1242$.

Sheline YI, Disabato BM, Hranilovich J, Morris C, D'Angelo G, Pieper C et al (2012). Treatment course with antidepressant therapy in late-life depression. Am J Psychiatry 169: $1185-1193$.

Sheline YI, Gado MH, Kraemer HC (2003). Untreated depression and hippocampal volume loss. Am J Psychiatry 160: $1516-1518$

Sheline YI, Wang PW, Gado MH, Csernansky JG, Vannier MW (1996). Hippocampal atrophy in recurrent major depression. Proc Natl Acad Sci USA 93: 3908-3913.

Spalletta G, Piras F, Caltagirone C, Fagioli S (2013). Hippocampal multimodal structural changes and subclinical depression in healthy individuals. J Affect Disord (e-pub ahead of print).

Stockmeier CA, Mahajan GJ, Konick LC, Overholser JC, Jurjus GJ, Meltzer HY et al (2004). Cellular changes in the postmortem hippocampus in major depression. Biol Psychiatry 56: 640-650.

Thomas AJ, Gallagher P, Robinson LJ, Porter RJ, Young AH, Ferrier IN et al (2009). A comparison of neurocognitive impairment in younger and older adults with major depression. Psychol Med 39: 725-733.

Trivedi MH, Fava M, Wisniewski SR, Thase ME, Quitkin F, Warden D et al (2006). Medication augmentation after the failure of SSRIs for depression. N Engl J Med 354: 1243-1252.

Trivedi MH, Rush AJ, Ibrahim HM, Carmody TJ, Biggs MM, Suppes T et al (2004). The Inventory of Depressive Symptomatology, Clinician Rating (IDS-C) and Self-Report (IDS-SR), and the Quick Inventory of Depressive Symptomatology, Clinician Rating (QIDS-C) and Self-Report (QIDS-SR) in public sector patients with mood disorders: a psychometric evaluation. Psychol Med 34: 73-82.

Turner RJ, Lloyd DA (2003). Cumulative adversity and drug dependence in young adults: racial/ethnic contrasts. Addiction 98: 305-315.

Vakili K, Pillay SS, Lafer B, Fava M, Renshaw PF, Bonello-Cintron $\mathrm{CM}$ et al (2000). Hippocampal volume in primary unipolar major depression: a magnetic resonance imaging study. Biol Psychiatry 47: 1087-1090.

Victor RG, Haley RW, Willett DL, Peshock RM, Vaeth PC, Leonard D et al (2004). The Dallas Heart Study: a population-based probability sample for the multidisciplinary study of ethnic differences in cardiovascular health. Am J Cardiol 93: 1473-1480.

Videbech P, Ravnkilde B (2004). Hippocampal volume and depression: a meta-analysis of MRI studies. Am J Psychiatry 161: 1957-1966. 